\title{
Pain is Highly Prevalent in Individuals with Tropical Spastic
}

\section{Paraparesis}

\author{
Selena Márcia Dubois Mendes ${ }^{1, *}$, Abrahão Fontes Baptista ${ }^{1,2,5, \#}$, Katia Nunes Sá ${ }^{1, \#}$, Daniel Ciampi de Araújo \\ Andrade $^{3}$, Grece Gomes Otero ${ }^{1}$, João Zugaib Cavalcanti ${ }^{1}$, Miúcha de Freitas Isensee ${ }^{1}$, Israel Souza ${ }^{4}$, Ramon \\ de Almeida Kruschewsky ${ }^{1}$, Bernardo Galvão-Castro, ${ }^{1,5, \#}$
}

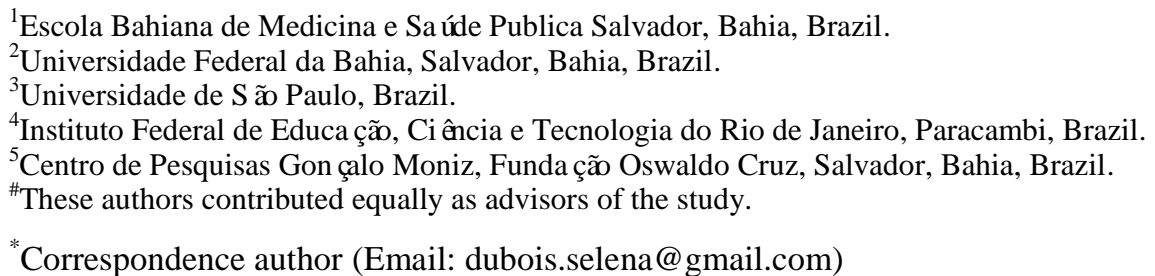

Abstract - To determine the prevalence and characteristics of pain in symptomatic and asymptomatic patients with HTLV-1, for HAM/TSP, Cross-sectional study conducted in patients with HTLV seen at a referral center for patient care and research in Salvador, Bahia, Brazil. Sociodemographic data were evaluated by means of interviews. The presence of neuropathic pain was evaluated by the DN4 questionnaire, its intensity by the Visual Analog Scale and its location by the body map, Among the 115 patients, $84.3 \%$ complained of pain. The most affected locations were the lumbar spine and lower limbs (63.1\%). Of these $46.1 \%$ presented defined or probable HAM/TSP. There was no association between the type of pain and presence of HAM/TSP, but symptomatic patients had a higher prevalence of pain in relation to asymptomatic for HAM/TSP, We found a high prevalence of pain in patients with HAM/TSP, mainly located in the lower back and lower limbs.

Keywords - Pain, Human T-lymphotropic virus 1, Paraparesis, Tropical Spastic, Myelitis

\section{Introduction}

The human T- cell lymphotropic virus type 1 (HTLV-1) may be etiologically linked with HTLV-1-associated myelopathy/ tropical spastic paraparesis (HAM/TSP), among other diseases (Poiesz et al. 1980; Gessain et al. 1985; Osame et al. 1986). It is estimated that 15 to 20 million people are infected with HTLV-1 worldwide but approximately $95 \%$ seem to remain asymptomatic. Japan, the Caribbean, Central and South America, Equatorial Africa, Middle East and Melanesia are the main endemic areas (Hlela et al. 2009). In Brazil it is estimated that 2.5 million people are living with HTLV-1, and the city of Salvador presents the highest prevalence in the country. A population-based study in this city demonstrated an overall prevalence of $1.74 \%$, which significantly increases, with age affecting $9 \%$ of women over the age of 51 years old. It was estimated that 40,000 people are infected by this virus (Dourado et al. 2003).

HAM/TSP is characterized as a demyelinating, slow and progressive chronic inflammatory disease of the central nervous system (Ijichi et al. 1995). The first manifestations of the disease occur mainly in the fourth decade of life, with a ratio of 2:1 female to male. Damage to the nervous system may induce motor-sensory disturbances, such as neuropathic pain and muscle weakness leading to gait disturbances and abnormal posture, which may induce nociceptive or mixed pain (Castro-Costa et al. 2009).

Pain has recently been shown as a major problem in HAM/TSP patients, but it is not still clear which type of pain is predominant (nociceptive/neuropathic) (Castro-Costa et al. 2009; Tavares et al. 2010; Netto \& Brites, 2011). Also, one could hypothesize that nociceptive pain is due to musculoskeletal abnormalities related to the disease, and neuropathic pain associated to lesion of nerve tissues in the spinal cord or peripheral nerves, but a specific pattern of pain before the manifestations of HAM/TSP was not described. This study sought to estimate the prevalence and to characterize the pain in HAM/TSP patients, compared to their counterpart control patients. 


\section{Material and Methods}

\subsection{Study design and population}

This is a cross-sectional descriptive study, conducted at Bahian School of Medicine and Public Health Reference Centre for HTLV in Salvador, Northeast Brazil. Data collection was carried out from June 2008 to July 2009. The diagnosis of HTLV-I infection was performed using ELISA (Cambridge Biotech Corp., Worcester, MA) and was confirmed by Western Blot analysis (HTLV blot 2.4, Genelab, Singapore) or Polymerase Chain Reaction (PCR). Co-infected patients with HIV and/or HCV were excluded.

Spinal cord nuclear magnetic resonance, serology for hepatitis $\mathrm{C}$ virus (HCV), syphilis, HIV and $\mathrm{HBV}$, fasting glucose, and vitamin B12 serum levels were assessed to exclude other potential causes of myelopathy (WHO Diagnostic Criteria). Myelopathic symptoms, serological findings, and/or detection of HTLV-1 DNA, as well as the exclusion of other disorders were used as indicators for diagnostic categorization according to ascertainment level (possible, probable and definite) (Castro-Costa et al. 2006). Patients with defined, probable and possible diagnostic for HAM/TSP were classified as symptomatic. Those ones infected by the virus without symptoms of myelopathy were considered asymptomatic.

One hundred and ninety-three individuals infected with HTLV-1 were interviewed of whom 78 patients not fulfilling the proposed diagnostic criteria, as well as those with cognitive deficits that impaired the understanding of the questionnaires were excluded from the study. Therefore, 115 individuals infected by HTLV-1, being 66 HAM/TSP (13 possible; 16 probable and 37definite) and 49 asymptomatic were included in the study.

\subsection{Data collection procedures and Instruments}

The data were collected in interviews by trained evaluators. The following instruments were used: 1) Sociodemographic and clinical data, obtained through a standardized questionnaire. Social class was defined by the ABIPEME criteria Associação Brasileira de Institutos de Pesquisa de Mercado (Brazilian Association of Market Research Institutes), which determines the distribution of the population by its class of income, considering the items of ownership and family comfort, with a classification from A (high income) to E (low income); 2) The Neuropathic Pain 4 Questionnaire (DN4) questionnaire translated and validated for Portuguese (Santos et al. 2010). The DN4 questionnaire discriminates between neuropathic and nociceptive. In this instrument, each location was evaluated separately. Patients responded whether there was the presence of pain with the characteristics of seven verbal pain descriptors, and there was a brief physical examination consisting of the three items assessed in the DN4 questionnaire. The presence of four or more descriptors/signs attested indicates the DN4 as being positive, and the presence of neuropathic pain; 3) Body Map (Diagram) was used for pain localization. The subject had to paint the areas of pain on a drawing of the human body, printed on a sheet of paper; 4) The Visual Analog Scale of Pain - VAS was used to evaluate pain intensity. This scale was in compliance with the categorization divided into slight ( 0 to 3 ), moderate (4 to 7 ) and intense (8 to 10$)$ pain.

\subsection{Data Analysis}

Sample characterization and diagnostic classification for HAM/TSP were generated by the description of frequency analysis. Univariate analysis of pain predictors, pain prevalence, associations between types of pain and diagnosis of HAM/TSP were estimated by the $G$ test. For data analysis of pain location and intensity, the grouping technique was used for both characteristics. An alpha value of 5\% was considered significant $(\mathrm{p}<0.05)$. The data were tabulated and analyzed in the Statistical Package for Social Science - SPSS (SPSS, Inc, Chicago, IL) version 14.0 for Windows.

\subsection{Statement of Ethics}

We certify that all applicable institutional and governmental regulations concerning the ethical use of human volunteers/animals were followed during the course of this research.

\section{Results}

Most of 115 individuals eligible for the study were women ( $n=80-69.6 \%$ ), age-range from 20 to 85 years old, with a mean age of $48.5 \pm 13.6$ years, and described themselves as mulatto $(40.0 \%)$ or black $(46.1 \%)$. The majority was married and belonged to D Social Class (59.1), with $>7$ years of schooling (54.8). The majority $(84.3 \%)$ of the individuals complained of some type of pain at the time of evaluation. In the univariate analysis of pain predictors in persons with HTLV-1, there was an association between pain and medical follow-up time $(\mathrm{G}=7.679, \mathrm{p}=0.021)$ with individuals who had been followed-up for longer than two years presenting higher prevalence $(>90.0 \%)$ (Table 1$)$.

The most affected locations were the lumbar spine and lower limbs. Analyses were performed taking into account all the pains mentioned, therefore the value extrapolates the number of individuals, as some individuals complained of more than one type of pain. The isolated pain locations were grouped into regions, in order to express their distribution more clearly. The region delimited by the head, face and neck accounted for 47 references $(7.19 \%)$; Thorax and abdominal regions, 27 references $(4.13 \%)$, region of the Upper Limbs, 167 references $(25.54 \%)$ and the Lumbar and Lower Limb regions, 413 references $(63.15 \%)$, in a total number of 654 references.

The patients were classified according to the number of pain locations reported, and it was verified that $12.4 \%$ complained of one to two pain locations, $35.1 \%$ from three to five locations, and $52.6 \%$ complained of six or more pain locations. As regards the intensity of the pains mentioned, $6.0 \% \quad(n=39)$ were slight, $45.0 \% \quad(n=292)$ moderate and $49.0 \% \quad(n=318)$ 
were intense. When only the maximum pain intensity of each patient was considered (as many of them had more than one site of pain), it was verified that $3.1 \%(n=3)$ had slight pain, $24.7 \%(n=24)$ moderate and $72.2 \%(n=70)$ had intense pain. When analyzing the association of data with reference to pain location and intensity, it was noted that in general, there was no preponderance of pain intensity with regard to its location $(\mathrm{G}=3.050 ; \mathrm{p}=0.803)$ (Table 2).

When the association of data with reference to pain loca- tion and type of pain (nociceptive or neuropathic) were analyzed, it was noted that there was an increase in neuropathic pain in the lumbar region and lower limbs (53.1\%), but this increase was not significant $(\mathrm{G}=6.764 ; \mathrm{p}=0.080)$ (Table 3).

When analyzing the association between the data referring to the type of pain and pain intensity, a preponderance of intense pain was noted in the neuropathic pain conditions $(\mathrm{G}=$ 45.908; $\mathrm{p}<0.001)$ (Table 3).

Table 1. Sociodemographic characteristics of sample and univariate analysis of predictors for pain in people with HTLV-1

\begin{tabular}{|c|c|c|c|}
\hline$n=115$ & $\begin{array}{c}\text { Sociodemographics } \\
\text { Characteristics } \\
\text { n }(\%)\end{array}$ & $\begin{array}{c}\text { Univariate Analysis of Pain } \\
\text { Predictors } \\
\text { n(\%;95\% CI })\end{array}$ & $\mathbf{p}$ \\
\hline Gender & & & $0.404^{(\mathrm{a})}$ \\
\hline Female & $80($ (69.6) & $69(86.3 ; 76.7-92.9)$ & \\
\hline Male & $35(30.4)$ & $28(80.0 ; 63.1-91.6)$ & \\
\hline Age & & & $0.524^{(b)}$ \\
\hline 20-34 years old & $17(14.8)$ & $13(76.5 ; 50.1-93.2)$ & \\
\hline $35-64$ years old & $83(72.2)$ & 72 (86.7; 77.5-93.2) & \\
\hline$\geq 65$ years old & $15(13.0)$ & $12(80.0 ; 51.9-95.7)$ & \\
\hline Conjugal Situation & & & $0.392^{(\mathrm{c})}$ \\
\hline Single & $40(35.1)$ & $31(77.5 ; 56.6-91.6)$ & \\
\hline Married & 47 (41.2) & $40(85.1 ; 71.7-93.8)$ & \\
\hline Separate/divorced & $15(13.2)$ & $14(93.3 ; 68.1-99.8)$ & \\
\hline Widower & $12(10.5)$ & $11(91.7 ; 61.5-99.8)$ & \\
\hline Self-reported skin color & & & $0.198^{(\mathrm{d})}$ \\
\hline White & $14(12.2)$ & $11(78.6 ; 49.2-95.3)$ & \\
\hline Black & $53(46.1)$ & $42(79.2 ; 65.9-89.2)$ & \\
\hline Mulatto & $46(40.0)$ & $42(91.3 ; 79.2-97.6)$ & \\
\hline Education (years) & & & $0.658^{(\mathrm{e})}$ \\
\hline$>7$ & $63(54.8)$ & $54(85.7 ; 74.6-93.3)$ & \\
\hline$\leq 7$ & $52(45.2)$ & 43 (82.7; 69.7-91.8) & \\
\hline Social class - Abipeme* & & & $0.154^{(\mathrm{f})}$ \\
\hline Class C & $23(20.0)$ & $17(73.9 ; 51.6-89.8)$ & \\
\hline Class D & $68(59.1)$ & 61 (89.7; 79.9-95.8) & \\
\hline Class E & $24(20.9)$ & $19(79.2 ; 57.9-92.9)$ & \\
\hline
\end{tabular}

(a)G=0.695; (b)G=1.291; (c) $\mathrm{G}=2.999 ;(\mathrm{d}) \mathrm{G}=3.238 ;$ (e) $\mathrm{G}=0.196 ;$ (f) $\mathrm{G}=3.737$; (g)G=7.679

* Associação Brasileira de Pesquisa de Mercado (Brazilian Association for Market Research) 
Table 2. Association between location and intensity of pain in HTLV-1 carriers

\begin{tabular}{lcccc}
\hline \multirow{2}{*}{ Location } & \multicolumn{3}{c}{ Intensity of pain, n (\%; CI95\%) } & \multirow{2}{*}{ Total } \\
\cline { 2 - 4 } & Slight & Moderate & Intense & \\
\hline $\begin{array}{l}\text { Head, face and } \\
\text { neck }\end{array}$ & $1(2.1 ; 0.1-11.3)$ & $25(53.2 ; 38.1-67.9)$ & $21(44.7 ; 30.2-59.9)$ & $47(7.2 ; 5.4-9.5)$ \\
Chest and belly & $2(7.4 ; 0.9-24.3)$ & $12(44.4 ; 25.5-64.7)$ & $13(48.1 ; 28.7-68.1)$ & $27(4.2 ; 2.8-6.0)$ \\
Upper limbs & $11(6.6 ; 3.3-11.5)$ & $77(46.1 ; 38.4-54.0)$ & $79(47.3 ; 39.5-55.2)$ & $167(25.7 ; 22.4-29.3)$ \\
$\begin{array}{l}\text { Lumbar and } \\
\text { lower limb }\end{array}$ & $25(6.1 ; 4.0-8.9)$ & $178(43.6 ; 38.6-48.6)$ & $205(50.2 ; 45.3-55.2)$ & $408(62.9 ; 59.0-66.6)$ \\
\hline Total & $39(6.0 ; 4.3-8.1)$ & $292(45.0 ; 41.1-48.9)$ & $318(49.0 ; 45.1-52.9)$ & $649(100.0)$ \\
\hline
\end{tabular}

$\mathrm{G}=3.050 ; \mathrm{p}=0.803$

Table 3. Association between location, intensity and type of pain in HTLV-1 carriers

\begin{tabular}{lccc}
\hline \multirow{2}{*}{ Location $^{(\text {(a) }}$} & \multicolumn{2}{c}{ Type of Pain, n (\%; CI95\%) } & Total \\
\cline { 2 - 3 } & Nociceptive Pain & Neuropathic Pain & \\
\hline Head, face and neck & $31(66.0 ; 50.7-79.1)$ & $16(34.0 ; 20.9-49.3)$ & $47(7.2 ; 5.4-9.5)$ \\
Chest and belly & $15(55.6 ; 35.3-74.5)$ & $12(44.4 ; 25.5-64.7)$ & $27(4.2 ; 2.8-6.0)$ \\
Upper limbs & $85(50.9 ; 43.1-58.7)$ & $82(49.1 ; 41.3-56.9)$ & $167(25.7 ; 22.4-29.2)$ \\
Lumbar and lower limbs & $192(46.9 ; 42.0-51.9)$ & $217(53.1 ; 48.1-58.0)$ & $409(62.9 ; 59.1-66.7)$ \\
Total & $\mathbf{3 2 3}(\mathbf{4 9 . 7} ; \mathbf{4 5 . 8 - 5 3 . 6})$ & $\mathbf{3 2 7}(\mathbf{5 0 . 3} ; \mathbf{4 6 . 4 - 5 4 . 2})$ & $\mathbf{6 5 0 ( 1 0 0 . 0 )}$ \\
\hline Intensity of pain ${ }^{(\mathbf{b})}$ & & & $39(6.0 ; 4.3-8.1)$ \\
\hline Slight & $23(59.0 ; 42.1-74.4)$ & $16(41.0 ; 25.6-57.9)$ & $292(45.0 ; 41.1-48.9)$ \\
Moderate & $184(63.0 ; 57.2-68.6)$ & $108(37.0 ; 31.4-42.8)$ & $318(49.0 ; 45.1-52.9)$ \\
Intense & $115(36.2 ; 30.9-41.7)$ & $203(63.8 ; 58.3-69.1)$ & $\mathbf{6 4 9}(\mathbf{1 0 0 . 0})$ \\
\hline Total & $\mathbf{3 2 2}(\mathbf{4 9 . 6} ; \mathbf{4 5 . 7 - 5 3 . 5})$ & $\mathbf{3 2 7}(\mathbf{5 0 . 4} ; \mathbf{4 6 . 5 - 5 4 . 3 )}$ & \\
\hline
\end{tabular}

(a): $G=6.764 ; p=0.080 ;(b): G=45.908 ; p<0.001$

Considering the classification for the diagnosis of HAM/TSP of Castro-Costa et al. (2006), the following distribution was found in the sample $(\mathrm{n}=115): 37(32.2 \%)$ defined, $16(13.9 \%)$ probable, $13(11.3 \%)$ possible, and $49(42.6 \%)$ asymptomatic. The prevalence of pain according to the diagnosis of HAM/TSP was $84.3 \%$ for the total sample, being $35 \%$ for the defined diagnosis; $16 \%$ for the probable; $12 \%$ for the possible, and $37 \%$ for the asymptomatic diagnosis. The analysis of this distribution associated with the prevalence of pain showed that in general, there was no preponderance of pain with regard to the diagnosis of HAM/TSP $(\mathrm{G}=7.735 ; \mathrm{p}=0.052)$ (Table 4). Nevertheless, when distributing the sample into the following groups: a) defined and probable HAM/TSP patients $(n=53)$ by excluding other neurologic conditions similar to this disease; $b$ ) possible ( $\mathrm{n}=13)$; and c) asymptomatic $(\mathrm{n}=49)$ patients, there was an association between the groups and types of pain; in the patients diagnosed as asymptomatic the proportion of the pain differs from that of the others $(\mathrm{G}=7.678 ; \mathrm{p}=0.022)$, being lower (73\% against 92\%) (Table 4$)$. When the same analysis was performed, however, separating the type of pain (none, neuropathic, nociceptive or mixed), once again, there was no association $(\mathrm{G}=11.962 ; \mathrm{p}=0.216)$, illustrating that the diagnosis of HAM/TSP did not determine which type of pain is most prevalent. Even when distributing the sample into defined and probable $(n=53)$; possible $(n=13)$ and asymptomatic $(n=49)$ groups, there was no association between the groups and the type of pain $(\mathrm{G}=10.108, \mathrm{p}=0.120)$ (Table 5). 
Table 4. Association of pain prevalence and diagnosis for HAM/TSP

\begin{tabular}{lccc}
\hline \multirow{2}{*}{ Diagnosis of HAM/TSP ${ }^{(a)}$} & \multicolumn{2}{c}{ Pain presence } & Total \\
\cline { 2 - 4 } & Without pain & With pain & $37(32.2 ; 23.8-41.5)$ \\
\hline Defined & $3(8.1 ; 1.7-2.2)$ & $34(91.9 ; 74.6-97.0)$ & $16(13.9 ; 8.2-21.6)$ \\
Probable & $1(6.3 ; 0.2-30.2)$ & $15(93.8 ; 69.8-99.8)$ & $13(11.3 ; 6.2-18.6)$ \\
Possible & $1(7.7 ; 0.2-36.0)$ & $12(92.3 ; 64.0-99.8)$ & $49(42.6 ; 33.4-52.2)$ \\
Asymptomatic & $13(26.5 ; 15.0-41.2)$ & $36(73.5 ; 58.9-85.1)$ & $115(100.0)$ \\
\hline Total & $18(15.7 ; 9.6-23.6)$ & $97(84.3 ; 76.4-90.5)$ & $53(46.1 ; 36.8-55.6)$ \\
\hline Diagnosis of HAM/TSP ${ }^{(b)}$ & $4(7.5 ; 2.1-18.2)$ & $49(92.5 ; 81.8-97.9)$ & $13(11.3 ; 6.2-18.6)$ \\
\hline Defined and probable & $1(7.7 ; 0.2-36.0)$ & $12(92.3 ; 64.0-99.8)$ & $49(42.6 ; 33.4-52.2)$ \\
Possible & $13(26.5 ; 15.0-41.1)$ & $36(73.5 ; 58.9-85.1)$ & $115(100.0)$ \\
Asymptomatic & $18(15.7 ; 10.0-23.6)$ & $97(84.3 ; 76.4-90.5)$ & \\
\hline Total & & & 4 \\
\hline
\end{tabular}

(a): $\mathrm{G}=7.735 ; \mathrm{p}=0.052 ;$ (b): $\mathrm{G}=7.678 ; \mathrm{p}=0.022$

Table 5. Association between diagnosis of HAM/TSP and type of pain

\begin{tabular}{|c|c|c|c|c|c|}
\hline \multirow{2}{*}{$\begin{array}{l}\text { Diagnosis of } \\
\text { HAM/TSP }^{(a)}\end{array}$} & \multicolumn{4}{|c|}{ Type of pain, n (\%; CI95\%) } & \multirow[t]{2}{*}{ Total } \\
\hline & None & Neuropathic & Nociceptive & Mixed & \\
\hline Probable & $1(6.3 ; 0.2-30.2)$ & $6(37.5 ; 15-64.6)$ & $3(18.8 ; 4.1-45.7)$ & $6(37.5 ; 15.2-64.6)$ & $\begin{array}{l}16(13.9 ; \\
8.2-21.6)\end{array}$ \\
\hline Possible & $1(7.7 ; 0.2-36.0)$ & $4(30.8 ; 9.1-61.4)$ & $3(23.1 ; 5.0-53.8)$ & $5(38.5 ; 13.9-68.4)$ & $\begin{array}{l}13(11.3 ; \\
6.2-18.6)\end{array}$ \\
\hline Asymptomatic & $13(26.5 ; 15.0-41.1)$ & $9(18.4 ; 8.8-32.0)$ & $16(32.7 ; 20.0-47.5)$ & $11(22.4 ; 11.8-36.6)$ & $\begin{array}{c}49(42.6 \\
33.4-52.2)\end{array}$ \\
\hline $\begin{array}{l}\text { Defined and } \\
\text { probable }\end{array}$ & $4(7.5 ; 2.1-18.2)$ & $14(26.4 ; 15.3-40.3)$ & $15(28.3 ; 16.8-42.4)$ & $20(37.7 ; 24.8-52.1)$ & $\begin{array}{c}53(46.1 ; \\
36.8-55.6)\end{array}$ \\
\hline Possible & $1(7.7 ; 0.2-36.0)$ & $4(30.8 ; 9.1-61.4)$ & $3(23.1 ; 5.0-53.8)$ & $5(38.5 ; 13.9-68.4)$ & $\begin{array}{l}13(11.3 ; \\
6.2-18.6)\end{array}$ \\
\hline Asymptomatic & $13(26.5 ; 15.0-41.1)$ & $9(18.4 ; 8.8-32.0)$ & $16(32.7 ; 20.0-47.5)$ & $11(22.4 ; 11.8-36.6)$ & $\begin{array}{c}49(42.6 \\
33.4-52.2)\end{array}$ \\
\hline Total & $18(15.7 ; 9.6-23.6)$ & $27(23.5 ; 16.1-32.3)$ & $34(29.6 ; 21.4-38.8)$ & $36(31.3 ; 23.0-40.6)$ & $115(100.0)$ \\
\hline
\end{tabular}

(a): $G=11.962 ; p=0.216 ;(b): G=10.108 ; p=0.120$

\section{Discussion}

In this study, a high prevalence of pain was verified in indi- viduals infected by the HTLV-1 $(84.3 \%, n=115)$. When the results of this study were extrapolated to the general population of this city, in which $41.4 \%$ suffer from chronic pain (Sá et al. 2008), there was evidence of the marked involvement of 
pain in the comorbidities associated with HTLV-1. Previous studies conducted in Brazil also found a high prevalence of pain in patients with HAM/TSP (Castro-Costa et al. 2009; Tavares et al. 2010; Netto \& Brites, 2011).

In the sample of the present study, there was no preponderance with regard to the prevalence of pain and the diagnostic classification of HAM/TSP, which was shown to be high for patients with defined, probable and possible HAM/TSP. Electrophysiological evidences in patients with HAM/TSP demonstrated nerve conduction deficits in motor-sensory pathways, at a peripheral and central level (Gessain et al. 1985). However, the spinal cord inflammatory process, characteristic of this infection, may be present before the symptomatology, which allows one to hypothesize it as being a trigger factor for pain in asymptomatic patients (Prommer et al. 2006).

The presence of pain in the asymptomatic patients can also be explained by sociodemographic factors already known in this population (Sá et al. 2008). The majority of women who were married and Afro-descendents belonged to low social class, educational level of less than seven years, middle-aged (35 to 64 years, corroborating previous studies conducted in Salvador (Dourado et al. 2003), other regions of Brazil (Proietti, Carneiro-Proietti, Catalan-Soares, \& Murphy, 2005; Araújo, \& Silva, 2006) and in other Latin American countries (Verdonck et al. 2007). The studied sample was exclusively composed of patients with a low socioeconomic level, which is related to a lower level of education, less access to information about prevention, and could therefore, elucidate the present findings. With reference to age, the results are in agreement with the data on the general population of this city, where there is a greater prevalence of adults; however, the results of this study may have presented this profile due to a predominance of volunteers in this age-range.

The most prevalent painful compromise in the lumbar spine and lower limbs found in this study is in agreement with previous studies (Castro-Costa et al. 2009; Tavares et al. 2010; Netto \& Brites, 2011; Proietti et al. 2005; Araújo et al. 2006; Verdonck et al. 2007), being justified by its neuropathology, with prominent inflammation of the inferior thoracic segments of the spinal cord (Franzoi et al. 2005). The clinical profile of HAM/TSP associated with HTLV-1 is compatible with the level of inflammatory lesions, and symptoms such as positive and negative sensitive phenomenon, spasticity / hyperreflexia (there may be clonus), and muscle weakness, mainly in the lower limbs, are outstanding (Ijichi et al. 1995). These characteristics may favor the onset of pain in the inferior half of the body; bearing in mind that muscle imbalance induces a joint instability that may possible lead to the injury of other tissues. Lumbar pain, per se, is an incapacitating factor for the HAM/TSP patient, because it promotes a lower level of activity, and consequently, a greater degree of deficiency (Franzoi et al. 2005).

When associating the data with reference to the location and the type of pain, a numerical, however, not statistical increase in neuropathic pain was observed in the lumbar regions and lower limbs. These data, is supported by previous studies that showed a statistical predominance of neuropathic pains (Castro-Costa et al. 2009; Netto \& Brites, 2011), and point out the need for a detailed clinical investigation to determine a therapeutic plan for these patients.

This study did not explore the tools that could direct investigations into urogynecological dysfunctions, which are an integral part of the clinical profile of these patients and could result in pain. It is possible that the body map was inefficient for use in analyzing this variable, in view of the extensive sphincter compromise presented, reflected as fewer neuralgic complaints in this region. This ratio suggests that the volunteers felt intimidated about filling in this part of the body map.

We hope that this study will provide visibility to the importance of pain in the HTLV infected patient. In future studies it could be verified whether there is an association with the pro-viral load, and what are the co-morbidities associated with HTLV-1 that could be related to these pain characteristics. Guidelines may be traced for a specific therapeutic approach to pain in the patient with this disease, bearing in mind the prevalence and the degree of incapacity produced.

This study concludes that there is a high prevalence of pain (84.3\%) in HTLV-1 patients. Pain was located mainly in the lumbar spine and lower limbs, with intensity ranging from moderate to severe. The type of pain did not depend on the diagnosis of HAM/TSP, but symptomatic patients presented higher prevalence of pain than asymptomatic to HAM/TSP. This panorama shows the need for further studies and public policies directed towards interdisciplinary attention in providing care of pain in the mentioned population.

\section{Acknowledgments}

The authors would like to thank the Bahian Foundation for the Development of Sciences for supporting this work.

\section{References}

Araújo, AQ-C., Silva, MTT. (2006). The HTLV-1 Neurological Complex. Lancet Neurol, 5, 1068-1076.

Castro-Costa, C. M., Araújo, AQ-C., Câmara, C. C., et al. (2009). Pain in tropical spastic paraparesis: HTLV-I associated myelopathy patients. Arq Neuro-Psiquiatr, 67(3-B), 866-870.

Castro-Costa, C. M., Araújo, AQ-C., Menna-Barreto, M., et al. (2006). Proposal for diagnostic criteria of tropical spastic paraparesis/HTLV-I-associated myelopathy (TSP/HAM). AIDS Res Hum Retroviruses, 22(10), 931-935.

Castillo, J. L., Cea, J. G., Verdugo, R. J., Cartier, L. (1999). Sensory Dysfunction in HTLV-1-Associeted Myelopathy / Tropical Spastic Paraparesis. Eur Neurol, 42, 17-22.

Dourado, I., Alcântara, L. C. J., Barreto, M. L.,Teixeira, M. G., Galvão-Castro, B. (2003). HTLV-I in the general population of Salvador, Brazil: a city with African ethnic and sociodemographic characteristics. J Acquir Immune Defic Syndr, 34(5), 527-531.

Franzoi, A. C., Araújo, AQ-C. (2005). Disability profile of patients with HTLV-1-associeted myelopathy / tropical spastic paraparesis using the Functional Independence Measure (FIMTM). Spinal Cord, 43, 236-240.

Gessain, A., Barin, F., Vernant, J. C., et al. (1985). Antibodies to human T-lymphotropic virus type-I in patients with tropical spastic paraparesis. Lancet, 8452, 407-410. 
Hlela, C., Shepperd, S., Khumalo, N. P., Taylor, G. P. (2009). The prevalence of human T-cell lymphotripic virus type 1 in the general population is unknown. AIDS Rev, 11, 205-214.

Ijichi, S., Osame, M. (1995). Human T Lymphotropic Virus Type I (HTLV) Associeted Myelopathy/Tropical Spastic Paraparesis (HAM/TSP) Recent Perspectives. Intern Med, 34, 713-721.

Netto, E. C., \& Brites, C. (2011). Characteristics of Chronic Pain and Its Impact on Quality of Life of Patients With HTLV-1-Associeted Myelopathy / Tropical Spastic Paraparesis (HAM/TSP) Clin J Pain, 27, 131-135.

Osame, M., Usuku, K., Izumo, S., Ijichi, N., Amitani, H., Igata, A., Matsumoto, M., Tara, M. (1986). HTLV-I associated myelopathy, a new clinical entity. Lancet. 1986 May 3;1(8488), 1031-2.

Poiesz, B. J., Ruscetti, F. W., Gazdar, A. F., Bunn, P. A., Minna, J. D., \& Gallo, R. C. (1980). Detection and isolation of type C retrovirus particles from fresh and cultured lymphocytes of a patient with cutaneous T-cell lymphoma. Proc Natl Acad Sci USA, 77, 7415-7419.
Prommer E. Ketamine and HTLV-1 Myelopathy: NMDA Blockade and Immunomodulation? J Pain Symptom Manage 2006, 31(5), 386-388.

Proietti, F. A., Carneiro-Proietti, A. B. (2005). Catalan-Soares, Murphy EL. Global epidemiology of HTLV-I infection and associated diseases. Oncogene, 24, 6058-6068.

Santos, J. G., Brito, J. O., de Andrade, D. C., et al. (2010). Translation to Portuquese and Validation of the Douleur Neuropathique 4 Questionnaire. J Pain, 11(5), 484-490.

Sá, K. N., Baptista, A. F., Matos, M. A., Lessa, I. (2008). Chronic Pain and Gender in Salvador Population, Brazil. Pain,139(3), 498-506.

Tavares, I. R., Franzoi, A. C., Araújo, AQ-C. (2010). Low-back pain in HTLV-I associated myelopathy / tropical spastic paraparesis: nociceptive or neuropathic? Spinal Cord, 48(2), 134-137;

Verdonck, K., González, E., Doreem, S. V., Vandamme, A-M., Vanham, Gotuzzo, E. (2007). Human T-lynphotropic virus 1: recent knowledge about an ancient infection, Lancet Infect Dis, 7, 266-281. 